\title{
DYNA3D Non-reflecting Boundary Conditions - Test Problems
}

Edward Zywicz

July 20, 2006 
This document was prepared as an account of work sponsored by an agency of the United States Government. Neither the United States Government nor the University of California nor any of their employees, makes any warranty, express or implied, or assumes any legal liability or responsibility for the accuracy, completeness, or usefulness of any information, apparatus, product, or process disclosed, or represents that its use would not infringe privately owned rights. Reference herein to any specific commercial product, process, or service by trade name, trademark, manufacturer, or otherwise, does not necessarily constitute or imply its endorsement, recommendation, or favoring by the United States Government or the University of California. The views and opinions of authors expressed herein do not necessarily state or reflect those of the United States Government or the University of California, and shall not be used for advertising or product endorsement purposes.

This work was performed under the auspices of the U.S. Department of Energy by University of California, Lawrence Livermore National Laboratory under Contract W-7405-Eng-48. 
UCRL-TR-224947

\title{
DYNA3D Non-reflecting Boundary Conditions - Test Problems
}

\author{
Edward Zywicz \\ Methods Development Group \\ Defense Technologies Engineering Division \\ Engineering Directorate
}

July 20, 2006

\section{SUMMARY}

Two verification problems were developed to test non-reflecting boundary segments in DYNA3D (Whirley and Engelmann, 1993). The problems simulate 1-D wave propagation in a semi-infinite rod using a finite length rod and non-reflecting boundary conditions. One problem examines pure pressure wave propagation, and the other problem explores pure shear wave propagation. In both problems the non-reflecting boundary segments yield results that differ only slightly (less than 6\%) during a short duration from their corresponding theoretical solutions. The errors appear to be due to the inability to generate a true step-function compressive wave in the pressure wave propagation problem and due to segment integration inaccuracies in the shear wave propagation problem. These problems serve as verification problems and as regression test problems for DYNA3D.

\section{THEORETICAL BASIS}

Under the assumption of small strain, isotropic, linear elasticity, Sokolnikoff (1986) presents the solution for wave propagation in an infinite solid. The solution is the combination of two distinct wave types - an equivoluminal shear wave and an irrotational pressure wave. The shear wave propagates at the velocity $c_{s}$, given by $c_{s}{ }^{2}=\mu / \rho$, and the pressure wave propagates at the velocity $c_{p}$, given by $c_{p}{ }^{2}=(\lambda+2 \mu) / \rho$. Here $\lambda$ is the Lame constant, $\mu$ is the shear modulus, and $\rho$ is the density.

When an incident acoustic wave encounters a material interface, a reflected wave and a transmitted wave are generated (Kinsler, et al, 1982). Continuity of traction requires that the sum of the incident and reflected wave tractions equals the transmitted wave traction along the interface. The magnitude of the reflected and transmitted waves depends upon the ratio of the impedance of the materials on each side of the boundary, where impedance is the ratio of the traction (pressure) and the particle velocity. The specific acoustic or pressure impedance is defined as $z_{p}=\rho c_{p}$ (Kinsler, 1982), while the specific shear impedance is defined here as $z_{s}=\rho c_{s}$. When the impedances are identical on both sides of the boundary, the transmitted wave is 
identical to the incident wave and no reflected wave is generated, i.e., the incident wave propagates across the interface without alteration.

DYNA3D's non-reflecting boundary conditions simulate the effect of a surrounding semiinfinite solid by imposing a traction, $\boldsymbol{t}$, along the designated boundary based upon impedance matching, i.e., the impedances of the material just outside the boundary are taken to be those of the material just inside the boundary. Thus, the imposed local traction used to simulate a nonreflecting boundary is given by

$$
\boldsymbol{t}=\left(\boldsymbol{l}(\boldsymbol{v} \bullet \boldsymbol{l})+\boldsymbol{m}\left(\boldsymbol{v}^{\bullet} \boldsymbol{m}\right)\right) z_{s}+\boldsymbol{n}\left(\boldsymbol{v}^{\bullet} \boldsymbol{n}\right) z_{n}
$$

where $\boldsymbol{v}$ is the velocity vector, $\boldsymbol{n}$ is the outward unit normal vector on the boundary, and $\boldsymbol{l}$ and $\boldsymbol{m}$ are unit tangential vectors that, along with $\boldsymbol{n}$, form a local orthogonal coordinate system. DYNA3D evaluates $\boldsymbol{t}$ at each non-reflecting boundary segment centroid and assumes it to be constant over the entire segment.

\section{TEST PROBLEM DESCRIPTIONS}

Two test problems were developed to verify DYNA3D's implementation of non-reflecting boundary conditions. The pressure and shear problems examine how non-reflecting boundary conditions handle a pure one-dimensional pressure wave and a pure one-dimensional shear wave, respectively. While the problem consists conceptually of a semi-infinite rod, the rod is numerically simulated as a long finite rod with non-reflecting boundary conditions imposed on its "infinite" end. An instantaneous normal or shear traction is imposed on the "free" end of the rod and held constant in time. The stresses along the rod are monitored to see if any reflected waves are generated.

\subsection{Pressure Wave Problem}

The pressure wave problem consists of a square rod 12 inches wide and high, and 600 inches long. The material has a Young's modulus of $3.338 \times 10^{6} \mathrm{psi}$, a zero Poisson's ratio, a mass density of 2.47x10-4 snail/inch ${ }^{3}$, and is simulated using DYNA3D's linear elastic material model 1. (A snail is a slug divided by 12 inches.). A pressure of 1/36 psi is imposed on the free end using concentrated nodal forces. Excluding the non-reflecting boundary segment on the end of the rod and the just-mentioned imposed pressure, there are no other imposed boundary

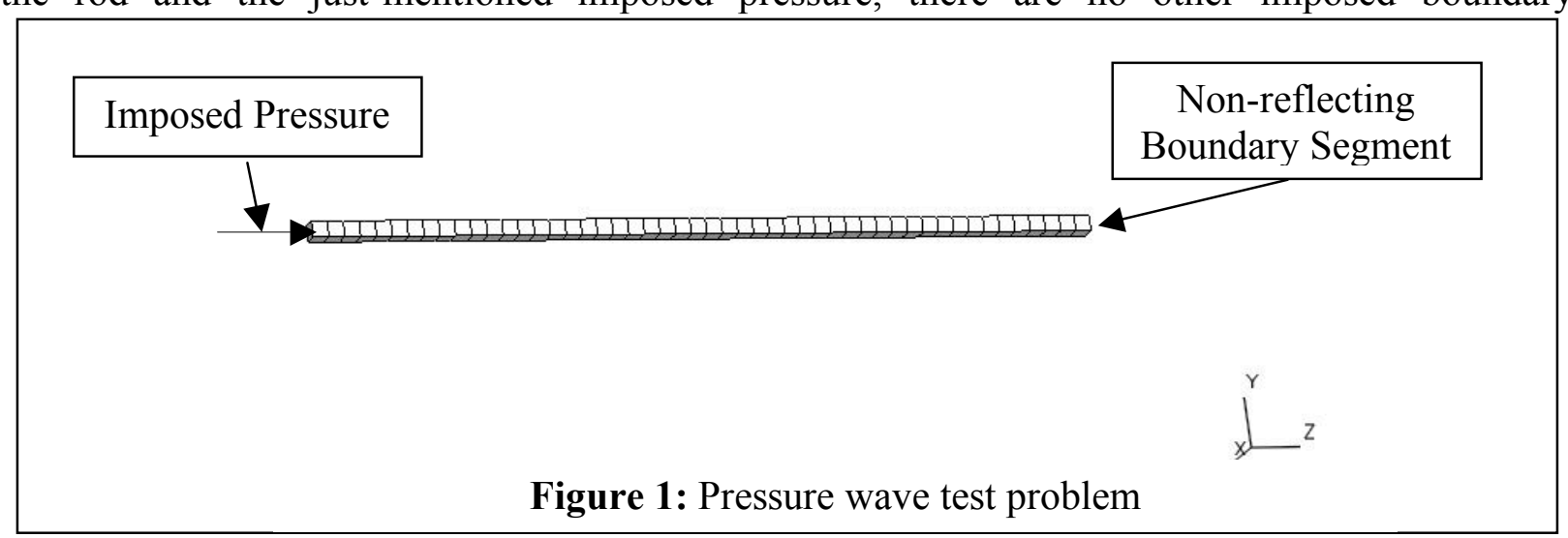


conditions. The problem is discretized, as shown in Figure 1, using 50 cubic hexahedral elements with exact volume, physical stabilization hourglass control. The simulation is run for $25 \mathrm{msec}$, or approximately 5 times the $5.16 \mathrm{msec}$ time it takes for a pressure wave to propagate the length of the rod. The elements whose centers are located 126, 246, 366, and 486 inches away from the free end (element numbers 10, 20, 30, and 40, respectively) are monitored throughout the simulation. Since the Poisson's ratio is zero, there is no lateral contraction as the wave propagates down the rod and the wave propagation is truly one-dimensional.

\subsection{Shear Wave Problem}

The shear wave problem consists of a square rod 96 inches wide and high, and 600 inches long. The material has the same mechanical properties as used in the pressure wave problem, but is simulated using DYNA3D's hyper-elastic compressible Mooney-Rivlin material model 27 (with $\mathrm{B}=0$ ). The problem is discretized using 3200 cubic hexahedral elements with exact volume, hyper-elastic physical stabilization hourglass control. As visible in Figure 2, there are 8 elements across the width and height, and 50 elements span the length. The first plane of 64 elements parallel to the free end of the rod is modeled as a rigid material. This facilitates the application of a $960 \mathrm{in}-\mathrm{lb}$ torque about the axis of the rod, imposed using two concentrated forces that generate the desired shear distribution. To ensure stability, the rigid material is restrained from rotating about either of its two other axes. The problem is run for $50 \mathrm{msec}$, or approximately 5 times the $10.32 \mathrm{msec}$ time it takes for a shear wave to propagate the length of the rod. The four surface elements that reside mid-side with centers located 126, 246, 366 and 486 inches away from the rigid-body end (element numbers $173,333,493$, and 653, respectively) are monitored throughout the simulation.

Since DYNA3D does not fully integrate the traction across the non-reflecting segment, a mesh convergence study was conducted. Naively, a mesh containing only one element across the height and width was tried. It resulted in a traction of $\boldsymbol{t}=\boldsymbol{0}$ being applied since the tangential velocity is zero at the segment's centroid. Needless to say, the non-reflecting boundary did not respond as desired. Various NxN cross-section discretizations were examined. Even with the current 8x8 mesh, some mesh-size-dependent errors are still apparent in the results.

\subsection{THEORETICAL RESPONSE}

The theoretical response is similar for both rods. In the pressure case, the only non-zero stress is the axial stress $\sigma_{\text {axial }}$, and its response is given by

$$
\sigma_{\text {axial }}=\sigma_{n} H\left(z-c_{p} t\right),
$$

where $\sigma_{n}$ is the applied stress $(0.027778 \mathrm{psi}), H$ is the Heavyside step function, $z$ is the distance from the free end, and $t$ is time. If the non-reflecting boundary is working correctly, there should be no wiggles or signs of any reflected waves in the response. In the shear case, it is intended that the only non-zero stresses are the two shear stresses on the axial face. Both of these shear components can be simultaneously monitored by examining the effective stress $\sigma_{\text {eff }}$, whose theoretical response for this problem is 
UCRL-TR-224947

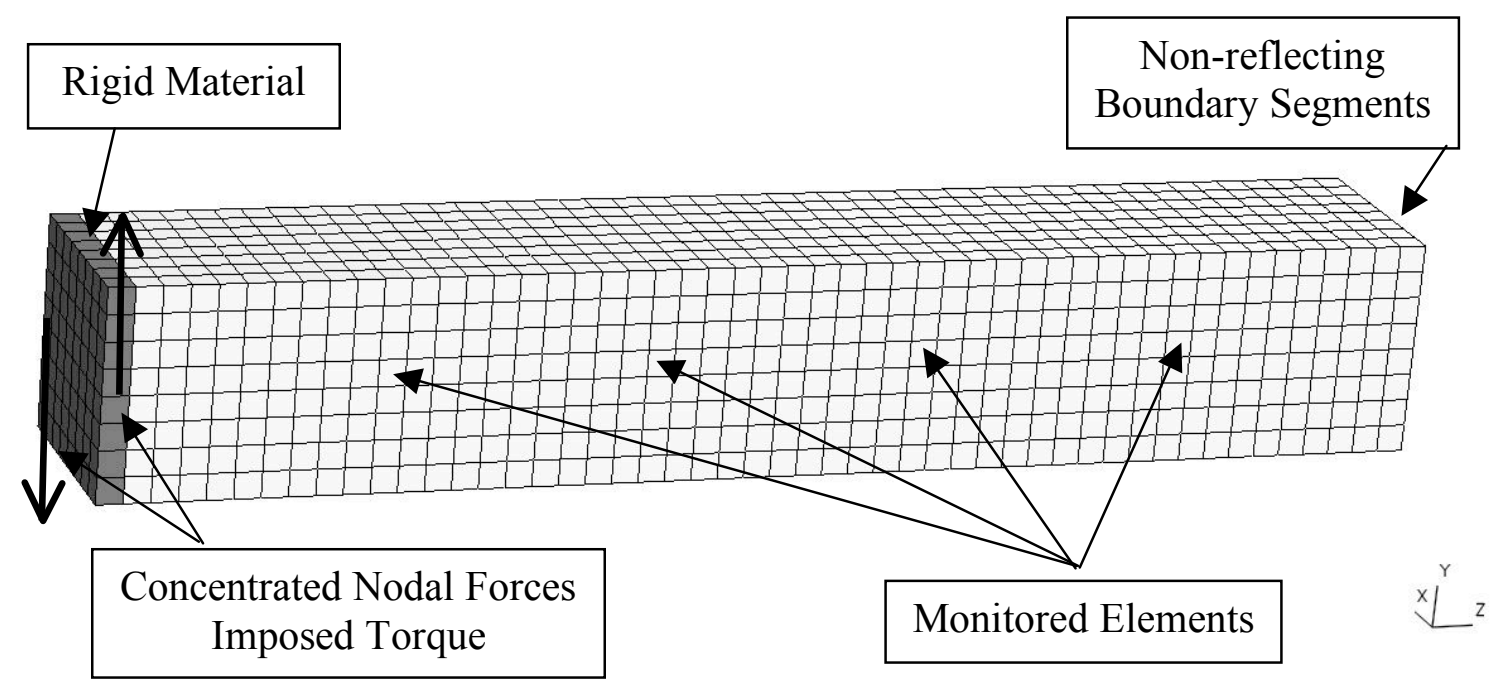

Figure 2: Shear wave test problem

$$
\sigma_{e f f}=\sigma_{r} H\left(z-c_{s} t\right) .
$$

Here $\sigma_{r}$ is a reference value that varies with the cross-section location and the applied torque magnitude. Unfortunately, square cross sections warp under torsion. Because axial displacements are precluded on the free end of the rod by the rigid material, some variations with axial position may occur in the stress field - especially near the ends.

\section{FE RESULTS}

\subsection{Pressure WaVe Problem}

Figure 3 shows the temporal response of the axial stress in the monitored elements for the pressure test case. Even with the bulk viscosity activated, there are some small oscillations in the compressive wave as it propagates down the rod during its initial traverse, i.e., the first 5.16 msec. After the initial wave reaches the non-reflecting end of the rod, a small "peak" in the stress response is visible. The peak, whose amplitude is approximately $6 \%$ of the nominal stress and duration is about $1 \mathrm{msec}$, emanates from the non-reflecting end, propagates back to the free-end, flips in sign, and then returns to the non-reflecting end before it disappears. Although not shown, the temporal responses of all the other stress components are essentially zero, i.e., numerical noise. With the exception of the small peak, the non-reflecting boundary appears to be functioning correctly and producing the theoretical and intended response. 
UCRL-TR-224947

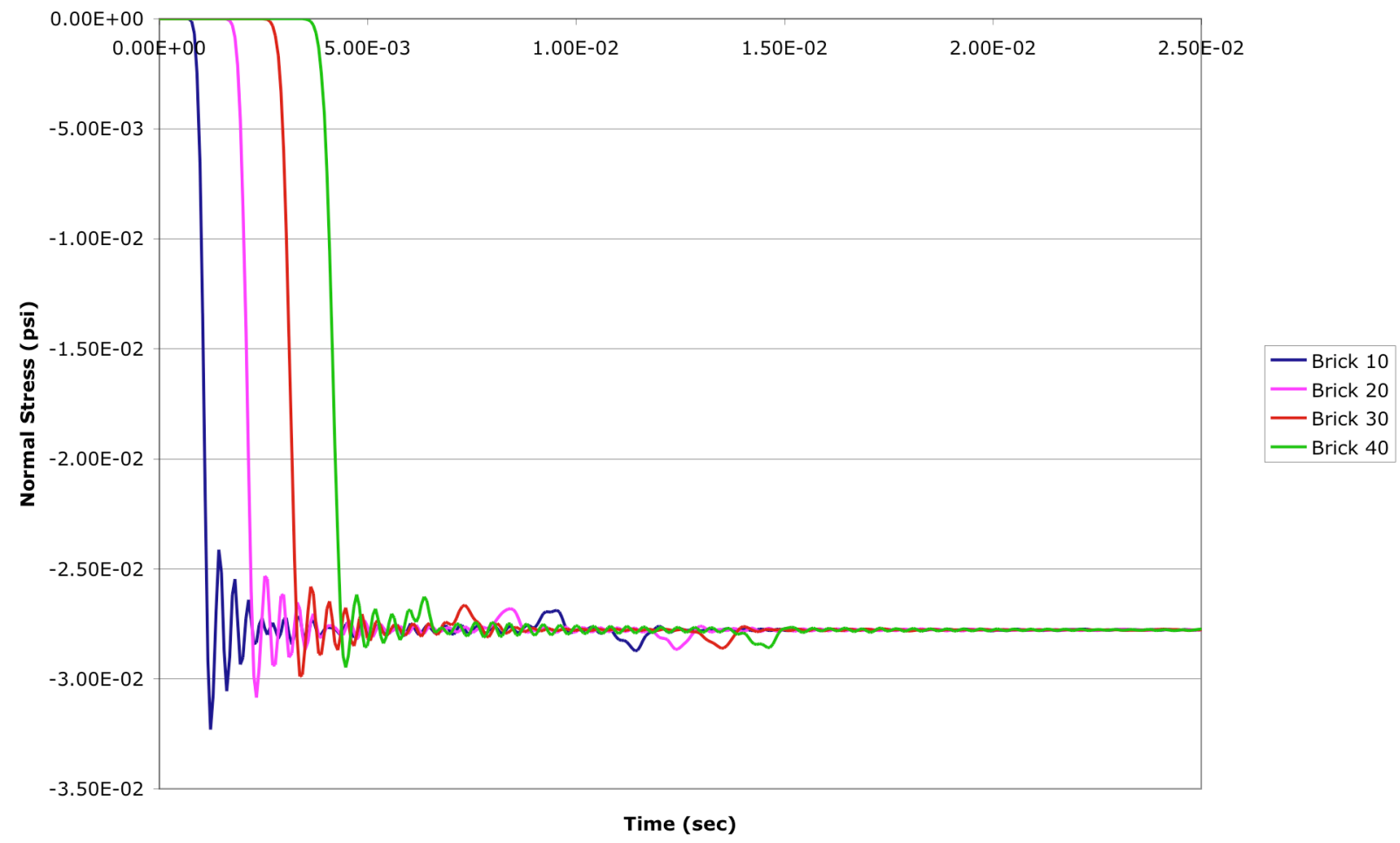

Figure 3: Axial stress versus time - pressure test problem

The pressure wave problem was also run without using artificial bulk viscosity. In this case, the incident wave contains considerable high frequency noise. The magnitude of the noise is about $18 \%$ of the mean value. Although some damping occurs over time, the stress response changes little as the initial wave reverberates along the rod. Based upon the behavior observed in this problem, it is assumed that the previously noted "peak" develops due to the non-constant initial incident wave and not due to a defect in the non-reflecting boundary condition development or implementation.

\subsection{Shear Wave Problem}

Figure 4 shows the temporal response of the effective stress in the monitored elements for the shear test case. As observed previously, there are some small oscillations in the wave profile as it propagates down the rod during its initial traverse, i.e., the first $10.32 \mathrm{msec}$. After the initial wave reaches the non-reflecting end of the rod, a small reflected wave is generated in the YZ shear component and this increases the effective stress by about $3.5 \%$. This wave, only present in the YZ shear component, propagates to the rigid end and then back to the non-reflecting end before it disappears. The response of the normal stress components and the XY shear component are similar to each other. Their signal consists of a small zero dwell period, then an initial rather sharp spike followed by high frequency noise of a smaller magnitude about zero. The magnitude of the spike and subsequent noise is approximately $2 \%$ and $0.2 \%$, respectively, of the mean effective stress $\left(7.35 \times 10^{-3} \mathrm{psi}\right)$. Overall, the square cross-section finite element solution agrees reasonably well with the round cross-section theoretical solution. 
UCRL-TR-224947

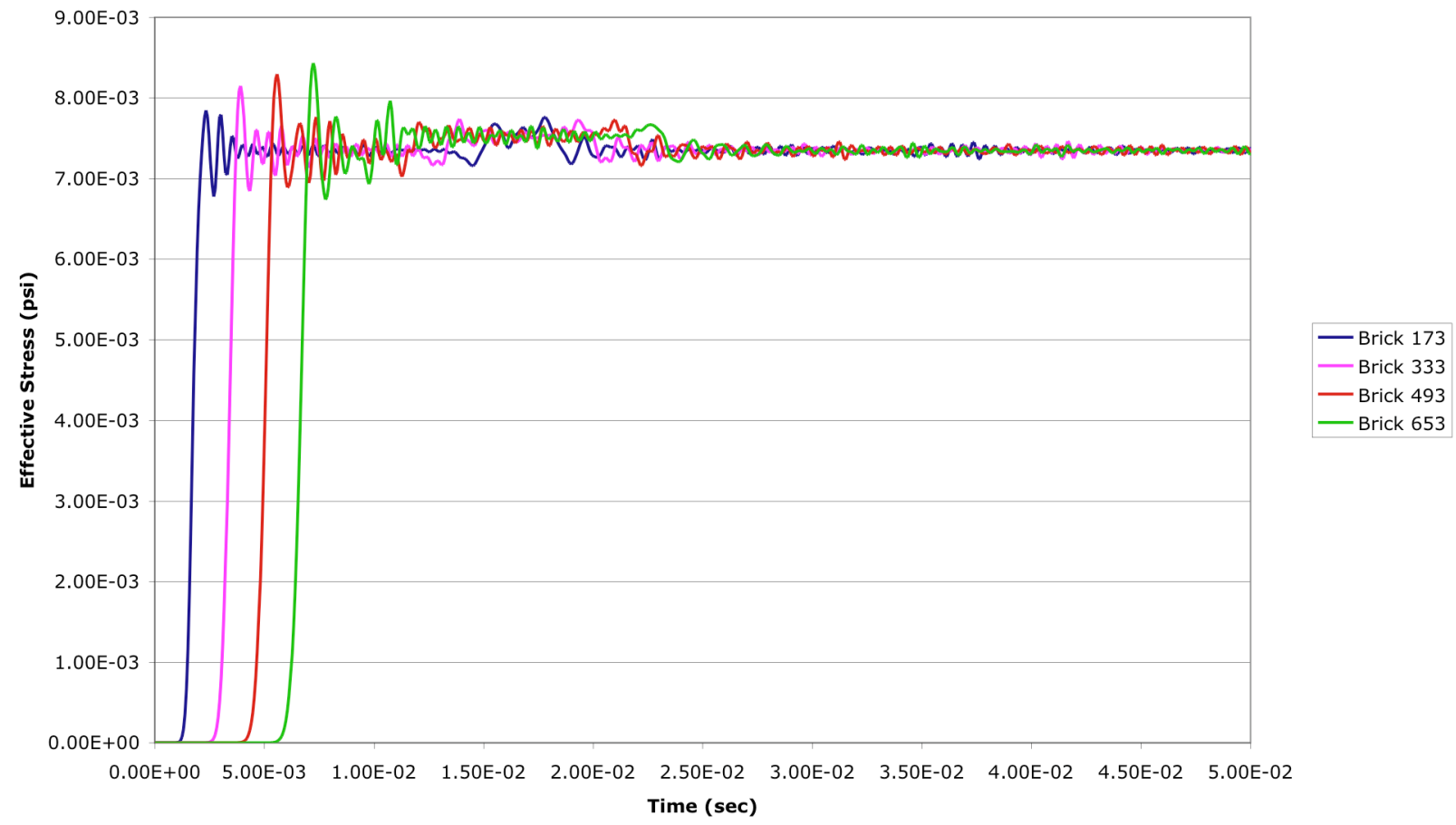

Figure 4: Effective stress versus time - shear test problem

To better understand the finite element result and ensure that the appropriate shear impedance was being used, the shear problem was rerun with the imposed shear impedance set to half its theoretical value. The effective stress profiles for this simulation are shown in Figure 5. While it is clear the non-reflecting boundary conditions are not doing their job, the wave reverberations are readily apparent. By examining Figures 4 and 5 together, there is no evidence of a pressure wave in the stress responses. (In this problem, the pressure wave velocity is twice the shear wave velocity.) Furthermore, the timing of the slight rise in stress seen in Figure 4 indicates it is some form of reflected wave. 
UCRL-TR-224947

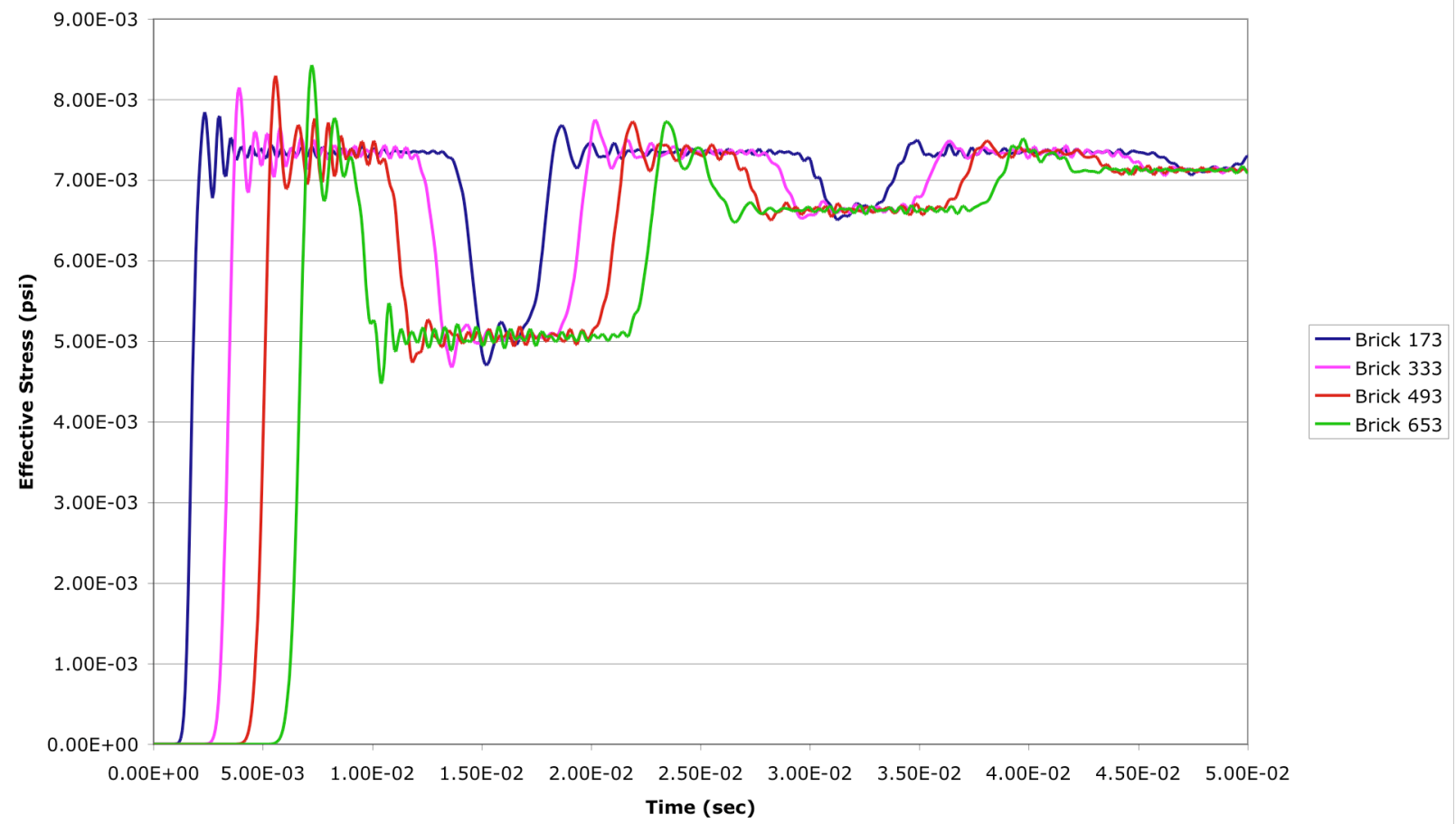

Figure 5: Effective stress versus time - shear test problem with modified shear impedance

The slight discrepancy between the square cross-section numerical solution and the round crosssection theoretical solution is presently assumed to be due to the single-point algorithm used to integrate the non-reflecting boundary segments. The fact that the discrepancy decreased as the cross-sectional mesh was refined supports this assumption. Nonetheless, the shear portion of the non-reflecting boundary condition appears to be implemented and functioning correctly.

\section{REGRESSION TESTING}

The test problems described above are included in DYNA3D's test suite as problems nrbc_norm and $n r b c_{-}$shear in the COUNT series. The appropriate stress component(s) at each of the four monitoring locations are written out, to four significant digits, 11 times during the simulation. The temporal stress responses are compared to stored values for regression testing purposes. The testing described in this report was done using DYNA3D, version 6.0, 7/18/2006, revision 1.901.

\section{ACKNOWLEDGEMENT}

Chad Noble originally developed the test problems described here. 
UCRL-TR-224947

\section{REFERENCES}

Kinsler, L.E., Frey, A.R., Coppens, A.B., and Sanders, J.V., Fundamentals of Acoustics, J. Wiley \& Sons, New York, 1982.

Sokolnikoff, I.S., Mathematical Theory of Elasticity, R. E. Krieger Publishing Company, Malabar, Florida, 1986.

Whirley, R.G., and Engelmann, B.E., "DYNA3D Users Manual", University of California, Lawrence Livermore National Laboratory, UCRL-MA-107254 Rev. 1, 1993. 